\title{
Insertion of a Contra-continuous Function between Two Comparable Real-valued Functions
}

\author{
Majid Mirmiran $^{1}$ and Binesh Naderi ${ }^{2}$ \\ ${ }^{1}$ Department of Mathematics, University of Isfahan, Isfahan 81746-73441, Iran \\ e-mail:mirmir@sci.ui.ac.ir \\ ${ }^{2}$ Department of General Courses, School of Management and Medical Information Sciences, \\ Isfahan University of Medical Sciences, Isfahan, Iran \\ e-mail: naderi@mng.mui.ac.ir
}

\begin{abstract}
A necessary and sufficient condition in terms of lower cut sets are given for the insertion of a contra-continuous function between two comparable real-valued functions on such topological spaces that kernel of sets are open.
\end{abstract}

\section{Introduction}

The concept of a $C$-open set in a topological space was introduced by Hatir et al. [12]. The authors define a set $S$ to be a $C$-open set if $S=U \cap A$, where $U$ is open and $A$ is semi-preclosed. A set $S$ is a $C$-closed set if its complement (denoted by $S^{c}$ ) is a $C$-open set or equivalently if $S=U \cup A$, where $U$ is closed and $A$ is semi-preopen. The authors show that a subset of a topological space is open if and only if it is an $\alpha$-open set and a $C$-open set or equivalently a subset of a topological space is closed if and only if it

Received: September 2, 2019; Accepted: October 22, 2019

2010 Mathematics Subject Classification: Primary 54C08, 54C10, 54C50; Secondary 26A15, 54C30.

Keywords and phrases: insertion, strong binary relation, $C$-open set, semi-preopen set, $\alpha$-open set, contracontinuous function, lower cut set.

This work was supported by University of Isfahan and Centre of Excellence for Mathematics (University of Isfahan).

Copyright (C) 2020 Majid Mirmiran and Binesh Naderi. This is an open access article distributed under the Creative Commons Attribution License, which permits unrestricted use, distribution, and reproduction in any medium, provided the original work is properly cited. 
is an $\alpha$-closed set and a $C$-closed set. This enables them to provide the following decomposition of continuity: a function is continuous if and only if it is $\alpha$-continuous and $C$-continuous or equivalently a function is contra-continuous if and only if it is contra- $\alpha$-continuous and contra- $C$-continuous.

Recall that a subset $A$ of a topological space $(X, \tau)$ is called $\alpha$-open if $A$ is the difference of an open and a nowhere dense subset of $X$. A set $A$ is called $\alpha$-closed if its complement is $\alpha$-open or equivalently if $A$ is the union of a closed and a nowhere dense set. Sets which are dense in some regular closed subspace are called semi-preopen or $\beta$-open. A set is semi-preclosed or $\beta$-closed if its complement is semi-preopen or $\beta$-open.

In [7] it was shown that a set $A$ is $\beta$-open if and only if $A \subseteq C l(\operatorname{Int}(C l(A)))$. A generalized class of closed sets was considered by Maki [19]. He investigated the sets that can be represented as union of closed sets and called them $V$-sets. Complements of $V$-sets, i.e., sets that are intersection of open sets are called $\Lambda$-sets [19].

Recall that a real-valued function $f$ defined on a topological space $X$ is called $A$-continuous [24] if the preimage of every open subset of $\mathbb{R}$ belongs to $A$, where $A$ is a collection of subsets of $X$. Most of the definitions of function used throughout this paper are consequences of the definition of A-continuity. However, for unknown concepts the reader may refer to $[4,11]$. In the recent literature many topologists had focused their research in the direction of investigating different types of generalized continuity.

Dontchev [5] introduced a new class of mappings called contra-continuity. Jafari and Noiri $[13,14]$ exhibited and studied among others a new weaker form of this class of mappings called contra- $\alpha$-continuous. A good number of researchers have also initiated different types of contra-continuous like mappings in the papers [1, 3, 8, 9, 10, 23].

Hence, a real-valued function $\mathrm{f}$ defined on a topological space $X$ is called contracontinuous (resp. contra-C-continuous, contra- $\alpha$-continuous) if the preimage of every open subset of $\mathbb{R}$ is closed (resp. $C$-closed, $\alpha$-closed) in $X$ [5].

Results of Katětov [15, 16] concerning binary relations and the concept of an indefinite lower cut set for a real-valued function, which is due to Brooks [2], are used in order to give a necessary and sufficient conditions for the insertion of a contracontinuous function between two comparable real-valued functions on such topological spaces that $\Lambda$-sets or kernel of sets are open [19]. 
If $g$ and $f$ are real-valued functions defined on a space $X$, we write $g \leq f$ (resp. $g<f$ ) in case $g(x) \leq f(x)$ (resp. $g(x)<f(x)$ ) for all $x$ in $X$.

The following definitions are modifications of conditions considered in [17].

A property $P$ defined relative to a real-valued function on a topological space is a cc-property provided that any constant function has property $P$ and provided that the sum of a function with property $P$ and any contra-continuous function also has property $P$. If $P_{1}$ and $P_{2}$ are $c c$-properties, the following terminology is used: (i) A space $X$ has the weak cc-insertion property for $\left(P_{1}, P_{2}\right)$ if and only if for any functions $g$ and $f$ on $X$ such that $g \leq f, g$ has property $P_{1}$ and $f$ has property $P_{2}$, then there exists a contracontinuous function $h$ such that $g \leq h \leq f$. (ii) A space $X$ has the $c c$-insertion property for $\left(P_{1}, P_{2}\right)$ if and only if for any functions $g$ and $f$ on $X$ such that $g<f, g$ has property $P_{1}$ and $f$ has property $P_{2}$, then there exists a contra-continuous function $h$ such that $g<h<f$. (iii) A space $X$ has the strong cc-insertion property for $\left(P_{1}, P_{2}\right)$ if and only if for any functions $g$ and $f$ on $X$ such that $g \leq f, g$ has property $P_{1}$ and $f$ has property $P_{2}$, then there exists a contra-continuous function $h$ such that $g \leq h \leq f$ and if $g(x)<f(x)$ for any $x$ in $X$, then $g(x)<h(x)<f(x)$. (iv) A space $X$ has the weakly cc-insertion property for $\left(P_{1}, P_{2}\right)$ if and only if for any functions $g$ and $f$ on $X$ such that $g<f, g$ has property $P_{1}, f$ has property $P_{2}$ and $f-g$ has property $P_{2}$, then there exists a contra-continuous function $h$ such that $g<h<f$.

In this paper, for a topological space whose $\Lambda$-sets or kernel of sets are open, is given a sufficient condition for the weak $c c$-insertion property. Also for a space with the weak $c c$-insertion property, we give a necessary and sufficient condition for the space to have the $c c$-insertion property. Several insertion theorems are obtained as corollaries of these results. In addition, the insertion and strong insertion of a contra- $\alpha$-continuous function between two comparable real-valued functions has also recently considered by the authors in $[20,21]$.

\section{The Main Result}

Before giving a sufficient condition for insertability of a contra-continuous function, the necessary definitions and terminology are stated. 
Definition 2.1. Let $A$ be a subset of a topological space $(X, \tau)$. We define the subsets $A^{\Lambda}$ and $A^{V}$ as follows:

$$
A^{\Lambda}=\bigcap\{O: O \supseteq A, O \in(X, \tau)\} \text { and } A^{V}=\bigcup\left\{F: F \subseteq A, F^{c} \in(X, \tau)\right\} .
$$

In [6, 18, 22], $A^{\Lambda}$ is called the kernel of $A$.

The family of all $\alpha$-open, $\alpha$-closed, $C$-open and $C$-closed will be denoted by $\alpha O(X, \tau), \alpha C(X, \tau), C O(X, \tau)$ and $C C(X, \tau)$, respectively.

We define the subsets $\alpha\left(A^{\Lambda}\right), \alpha\left(A^{V}\right), C\left(A^{\Lambda}\right)$ and $C\left(A^{V}\right)$ as follows:

$$
\begin{aligned}
& \alpha\left(A^{\Lambda}\right)=\bigcap\{O: O \supseteq A, O \in \alpha O(X, \tau)\}, \\
& \alpha\left(A^{V}\right)=\bigcup\{F: F \subseteq A, F \in \alpha C(X, \tau)\}, \\
& C\left(A^{\Lambda}\right)=\bigcap\{O: O \supseteq A, O \in C O(X, \tau)\} \text { and } \\
& C\left(A^{V}\right)=\bigcup\{F: F \subseteq A, F \in C C(X, \tau)\} . \\
& \alpha\left(A^{\Lambda}\right)\left(\text { resp. } C\left(A^{\Lambda}\right)\right) \text { is called the } \alpha \text {-kernel (resp. C-kernel) of } A .
\end{aligned}
$$

The following first two definitions are modifications of conditions considered in [15, $16]$.

Definition 2.2. If $\rho$ is a binary relation in a set $S$, then $\bar{\rho}$ is defined as follows: $x \bar{\rho} y$ if and only if $y \rho v$ implies $x \rho v$ and $u \rho x$ implies $u \rho y$ for any $u$ and $v$ in $S$.

Definition 2.3. A binary relation $\rho$ in the power set $P(X)$ of a topological space $X$ is called a strong binary relation in $P(X)$ in case $\rho$ satisfies each of the following conditions:

(1) If $A_{i} \rho B_{j}$ for any $i \in\{1, \ldots, m\}$ and for any $j \in\{1, \ldots, n\}$, then there exists a set $C$ in $P(X)$ such that $A_{i} \rho C$ and $C \rho B_{j}$ for any $i \in\{1, \ldots, m\}$ and any $j \in\{1, \ldots, n\}$.

(2) If $A \subseteq B$, then $A \bar{\rho} B$.

(3) If $A \rho B$, then $A^{\Lambda} \subseteq B$ and $A \subseteq B^{V}$. 
The concept of a lower indefinite cut set for a real-valued function was defined by Brooks [2] as follows:

Definition 2.4. If $f$ is a real-valued function defined on a space $X$ and if $\{x \in X: f(x)<\ell\} \subseteq A(f, \ell) \subseteq\{x \in X: f(x) \leq \ell\}$ for a real number $\ell$, then $A(f, \ell)$ is called a lower indefinite cut set in the domain of $f$ at the level $\ell$.

We now give the following main result:

Theorem 2.1. Let $g$ and $f$ be real-valued functions on the topological space $X$, in which kernel sets are open, with $g \leq f$. If there exists a strong binary relation $\rho$ on the power set of $X$ and if there exist lower indefinite cut sets $A(f, t)$ and $A(g, t)$ in the domain of $f$ and $g$ at the level $t$ for each rational number $t$ such that if $t_{1}<t_{2}$, then $A\left(f, t_{1}\right) \rho A\left(g, t_{2}\right)$, then there exists a contra-continuous function $h$ defined on $X$ such that $g \leq h \leq f$.

Proof. Let $g$ and $f$ be real-valued functions defined on the $X$ such that $g \leq f$. By hypothesis there exists a strong binary relation $\rho$ on the power set of $X$ and there exist lower indefinite cut sets $A(f, t)$ and $A(g, t)$ in the domain of $f$ and $g$ at the level $t$ for each rational number $t$ such that if $t_{1}<t_{2}$, then $A\left(f, t_{1}\right) \rho A\left(g, t_{2}\right)$.

Define functions $F$ and $G$ mapping the rational numbers $\mathbb{Q}$ into the power set of $X$ by $F(t)=A(f, t)$ and $G(t)=A(g, t)$. If $t_{1}$ and $t_{2}$ are any elements of $\mathbb{Q}$ with $t_{1}<t_{2}$, then $F\left(t_{1}\right) \bar{\rho} F\left(t_{2}\right), G\left(t_{1}\right) \bar{\rho} G\left(t_{2}\right)$, and $F\left(t_{1}\right) \rho G\left(t_{2}\right)$. By Lemmas 1 and 2 in [16] it follows that there exists a function $H$ mapping $\mathbb{Q}$ into the power set of $X$ such that if $t_{1}$ and $t_{2}$ are any rational numbers with $t_{1}<t_{2}$, then $F\left(t_{1}\right) \rho H\left(t_{2}\right), H\left(t_{1}\right) \rho H\left(t_{2}\right)$ and $H\left(t_{1}\right) \rho G\left(t_{2}\right)$.

For any $x$ in $X$, let $h(x)=\inf \{t \in \mathbb{Q}: x \in H(t)\}$.

We first verify that $g \leq h \leq f$ : If $x$ is in $H(t)$, then $x$ is in $G\left(t^{\prime}\right)$ for any $t^{\prime}>t$; since $x$ is in $G\left(t^{\prime}\right)=A\left(g, t^{\prime}\right)$ implies that $g(x) \leq t^{\prime}$, it follows that $g(x) \leq t$. Hence $g \leq h$. If $x$ is not in $H(t)$, then $x$ is not in $F\left(t^{\prime}\right)$ for any $t^{\prime}<t$; since $x$ is not in $F\left(t^{\prime}\right)=A\left(f, t^{\prime}\right)$ implies that $f(x)>t^{\prime}$, it follows that $f(x) \geq t$. Hence $h \leq f$.

Also, for any rational numbers $t_{1}$ and $t_{2}$ with $t_{1}<t_{2}$, we have $h^{-1}\left(t_{1}, t_{2}\right)=$ 
$H\left(t_{2}\right)^{V} \backslash H\left(t_{1}\right)^{\Lambda}$. Hence $h^{-1}\left(t_{1}, t_{2}\right)$ is closed in $X$, i.e., $h$ is a contra-continuous function on $X$.

The above proof used the technique of Theorem 1 in [15].

Theorem 2.2. Let $P_{1}$ and $P_{2}$ be cc-property and $X$ be a space that satisfies the weak cc-insertion property for $\left(P_{1}, P_{2}\right)$. Also assume that $g$ and $f$ are functions on $X$ such that $g<f, g$ has property $P_{1}$ and $f$ has property $P_{2}$. The space $X$ has the cc-insertion property for $\left(P_{1}, P_{2}\right)$ if and only if there exist lower cut sets $A\left(f-g, 3^{-n+1}\right)$ and there exists a decreasing sequence $\left\{D_{n}\right\}$ of subsets of $X$ with empty intersection and such that for each $n, X \backslash D_{n}$ and $A\left(f-g, 3^{-n+1}\right)$ are completely separated by contra-continuous functions.

Proof. Assume that $X$ has the weak $c c$-insertion property for $\left(P_{1}, P_{2}\right)$. Let $g$ and $f$ be functions such that $g<f, g$ has property $P_{1}$ and $f$ has property $P_{2}$. By hypothesis there exist lower cut sets $A\left(f-g, 3^{-n+1}\right)$ and there exists a sequence $\left(D_{n}\right)$ such that $\bigcap_{n=1}^{\infty} D_{n}=\varnothing$ and such that for each $n, X \backslash D_{n}$ and $A\left(f-g, 3^{-n+1}\right)$ are completely separated by contra-continuous functions. Let $k_{n}$ be a contra-continuous function such that $k_{n}=0$ on $A\left(f-g, 3^{-n+1}\right)$ and $k_{n}=1$ on $X \backslash D_{n}$. Let a function $k$ on $X$ be defined by

$$
k(x)=1 / 2 \sum_{n=1}^{\infty} 3^{-n} k_{n}(x) .
$$

By the Cauchy condition and the properties contra-continuous functions, the function $k$ is a contra-continuous function. Since $\bigcap_{n=1}^{\infty} D_{n}=\varnothing$ and since $k_{n}=1$ on $X \backslash D_{n}$, it follows that $0<k$. Also $2 k<f-g$ : In order to see this, observe first that if $x$ is in $A\left(f-g, 3^{-n+1}\right)$, then $k(x) \leq 1 / 4\left(3^{-n}\right)$. If $x$ is any point in $X$, then $x \notin(f-g, 1)$ or for some $n$,

$$
x \in A\left(f-g, 3^{-n+1}\right)-A\left(f-g, 3^{-n}\right)
$$

in the former case $2 k(x)<1$, and in the latter $2 k(x) \leq 1 / 2\left(3^{-n}\right)<f(x)-g(x)$. Thus if 
$f_{1}=f-k$ and if $g_{1}=g+k$, then $g<g_{1}<f_{1}<f$. Since $P_{1}$ and $P_{2}$ are E-properties, then $g_{1}$ has property $P_{1}$ and $f_{1}$ has property $P_{2}$. Since $X$ has the weak $c c$-insertion property for $\left(P_{1}, P_{2}\right)$, then there exists a contra-continuous function $h$ such that $g_{1} \leq h \leq f_{1}$. Thus $g<h<f$, it follows that $X$ satisfies the $c c$-insertion property for $\left(P_{1}, P_{2}\right)$. (The technique of this proof is by Katětov [15]).

Conversely, let $g$ and $f$ be functions on $X$ such that $g$ has property $P_{1}, f$ has property $P_{2}$ and $g<f$. By hypothesis, there exists a contra-continuous function $h$ such that $g<h<f$. We follow an idea contained in Lane [17]. Since the constant function 0 has property $P_{1}$, since $f-h$ has property $P_{2}$, and since $X$ has the $c c$-insertion property for $\left(P_{1}, P_{2}\right)$, then there exists a contra-continuous function $k$ such that $0<k<f-h$. Let $A\left(f-g, 3^{-n+1}\right)$ be any lower cut set for $f-g$ and let $D_{n}=\left\{x \in X: k(x)<3^{-n+2}\right\}$. Since $k>0$ it follows that $\bigcap_{n=1}^{\infty} D_{n}=\varnothing$. Since

$$
A\left(f-g, 3^{-n+1}\right) \subseteq\left\{x \in X:(f-g)(x) \leq 3^{-n+1}\right\} \subseteq\left\{x \in X: k(x) \leq 3^{-n+1}\right\}
$$

and since $\left\{x \in X: k(x) \leq 3^{-n+1}\right\}$ and $\left\{x \in X: k(x) \geq 3^{-n+2}\right\}=X \backslash D_{n}$ are completely separated by contra-continuous functions $\sup \left\{3^{-n+1}, \inf \left\{k, 3^{-n+2}\right\}\right.$, it follows that for each $n, A\left(f-g, 3^{-n+1}\right)$ and $X \backslash D_{n}$ are completely separated by contra-continuous functions.

\section{Applications}

The abbreviations $c \alpha c$ and $c C c$ are used for contra- $\alpha$-continuous and contra- $C$ continuous, respectively.

Before stating the consequences of Theorems 2.1, 2.2, we suppose that $X$ is a topological space whose kernel sets are open.

Corollary 3.1. If for each pair of disjoint $\alpha$-open (resp. C-open) sets $G_{1}, G_{2}$ of $X$, there exist closed sets $F_{1}$ and $F_{2}$ of $X$ such that $G_{1} \subseteq F_{1}, G_{2} \subseteq F_{2}$ and $F_{1} \cap F_{2}=\varnothing$, then $X$ has the weak cc-insertion property for $(c \alpha c, c \alpha c)$ (resp. $(c C c, c C c))$.

Proof. Let $g$ and $f$ be real-valued functions defined on $X$, such that $f$ and $g$ are $c \alpha c$ 
(resp. $c C c$ ), and $g \leq f$. If a binary relation $\rho$ is defined by $A \rho B$ in case $\alpha\left(A^{\Lambda}\right) \subseteq \alpha\left(B^{V}\right) \quad\left(\right.$ resp. $\left.C\left(A^{\Lambda}\right) \subseteq C\left(B^{V}\right)\right)$, then by hypothesis $\rho$ is a strong binary relation in the power set of $X$. If $t_{1}$ and $t_{2}$ are any elements of $\mathbb{Q}$ with $t_{1}<t_{2}$, then

$$
A\left(f, t_{1}\right) \subseteq\left\{x \in X: f(x) \leq t_{1}\right\} \subseteq\left\{x \in X: g(x)<t_{2}\right\} \subseteq A\left(g, t_{2}\right) ;
$$

since $\left\{x \in X: f(x) \leq t_{1}\right\}$ is an $\alpha$-open (resp. $C$-open) set and since $\left\{x \in X: g(x)<t_{2}\right\}$ is an $\alpha$-closed (resp. $C$-closed) set, it follows that $\alpha\left(A\left(f, t_{1}\right)^{\Lambda}\right) \subseteq \alpha\left(A\left(g, t_{2}\right)^{V}\right)$ (resp. $\left.C\left(A\left(f, t_{1}\right)^{\Lambda}\right) \subseteq C\left(A\left(g, t_{2}\right)^{V}\right)\right)$. Hence $t_{1}<t_{2}$ implies that $A\left(f, t_{1}\right) \rho A\left(g, t_{2}\right)$. The proof follows from Theorem 2.1.

Corollary 3.2. If for each pair of disjoint $\alpha$-open (resp. C-open) sets $G_{1}, G_{2}$ there exist closed sets $F_{1}$ and $F_{2}$ such that $G_{1} \subseteq F_{1}, G_{2} \subseteq F_{2}$ and $F_{1} \cap F_{2}=\varnothing$, then every contra- $\alpha$-continuous (resp. contra-C-continuous) function is contra-continuous.

Proof. Let $f$ be a real-valued contra- $\alpha$-continuous (resp. contra- $C$-continuous) function defined on $X$. Set $g=f$, then by Corollary 3.1, there exists a contracontinuous function $h$ such that $g=h=f$.

Corollary 3.3. If for each pair of disjoint $\alpha$-open (resp. C-open) sets $G_{1}, G_{2}$ of $X$, there exist closed sets $F_{1}$ and $F_{2}$ of $X$ such that $G_{1} \subseteq F_{1}, G_{2} \subseteq F_{2}$ and $F_{1} \cap F_{2}=\varnothing$, then $X$ has the strong cc-insertion property for $(\mathrm{c} \alpha \mathrm{c}, \mathrm{c} \alpha \mathrm{c})(\mathrm{resp} .(c C c, c C c))$.

Proof. Let $g$ and $f$ be real-valued functions defined on the $X$, such that $f$ and $g$ are $c \alpha c$ (resp. $c C c$ ), and $g \leq f$. Set $h=(f+g) / 2$, thus $g \leq h \leq f$ and if $g(x)<f(x)$ for any $x$ in $X$, then $g(x)<h(x)<f(x)$. Also, by Corollary 3.2, since $g$ and $f$ are contracontinuous functions hence $h$ is a contra-continuous function.

Corollary 3.4. If for each pair of disjoint subsets $G_{1}, G_{2}$ of $X$, such that $G_{1}$ is $\alpha$-open and $G_{2}$ is $C$-open, there exist closed subsets $F_{1}$ and $F_{2}$ of $X$ such that $G_{1} \subseteq F_{1}$, $G_{2} \subseteq F_{2}$ and $F_{1} \cap F_{2}=\varnothing$, then $X$ have the weak cc-insertion property for $(c \alpha c, c C c)$ and $(c C c, c \alpha c)$.

Proof. Let $g$ and $f$ be real-valued functions defined on $X$, such that $g$ is $c \alpha c$ (resp. 
$c C c$ ) and $f$ is $c C c$ (resp. $c \alpha c$ ), with $g \leq f$. If a binary relation $\rho$ is defined by $A \rho B$ in case $C\left(A^{\Lambda}\right) \subseteq \alpha\left(B^{V}\right)$ (resp. $\left.\alpha\left(A^{\Lambda}\right) \subseteq C\left(B^{V}\right)\right)$, then by hypothesis $\rho$ is a strong binary relation in the power set of $X$. If $t_{1}$ and $t_{2}$ are any elements of $\mathbb{Q}$ with $t_{1} \leq t_{2}$, then

$$
A\left(f, t_{1}\right) \subseteq\left\{x \in X: f(x) \leq t_{1}\right\} \subseteq\left\{x \in X: g(x)<t_{2}\right\} \subseteq A\left(g, t_{2}\right) ;
$$

since $\left\{x \in X: f(x) \leq t_{1}\right\}$ is a $C$-open (resp. $\alpha$-open) set and since $\left\{x \in X: g(x) \leq t_{2}\right\}$ is an $\alpha$-closed (resp. $C$-closed) set, it follows that $C\left(A\left(f, t_{1}\right)^{\Lambda}\right) \subseteq \alpha\left(A\left(g, t_{2}\right)^{V}\right)$ (resp. $\alpha\left(A\left(f, t_{1}\right)^{\Lambda}\right) \subseteq C\left(A\left(g, t_{2}\right)^{V}\right)$. Hence $t_{1} \leq t_{2}$ implies that $A\left(f, t_{1}\right) \rho A\left(g, t_{2}\right)$. The proof follows from Theorem 2.1.

Before stating consequences of Theorem 2.2, we state and prove the necessary lemmas.

Lemma 3.1. The following conditions on the space $X$ are equivalent:

(i) For each pair of disjoint subsets $G_{1}, G_{2}$ of $X$, such that $G_{1}$ is $\alpha$-open and $G_{2}$ is $C$-open, there exist closed subsets $F_{1}, F_{2}$ of $X$ such that $G_{1} \subseteq F_{1}, G_{2} \subseteq F_{2}$ and $F_{1} \cap F_{2}=\varnothing$.

(ii) If $G$ is a $C$-open (resp. $\alpha$-open) subset of $X$ which is contained in an $\alpha$-closed (resp. $C$-closed) subset $F$ of $X$, then there exists a closed subset $H$ of $X$ such that $G \subseteq H \subseteq H^{\Lambda} \subseteq F$.

Proof. (i) $\Rightarrow$ (ii) Suppose that $G \subseteq F$, where $G$ and $F$ are $C$-open (resp. $\alpha$-open) and $\alpha$-closed (resp. $C$-closed) subsets of $X$, respectively. Hence, $F^{c}$ is an $\alpha$-open (resp. $C$-open) and $G \cap F^{c}=\varnothing$.

By (i) there exists two disjoint closed subsets $F_{1}, F_{2}$ such that $G \subseteq F_{1}$ and $F^{c} \subseteq F_{2}$. But

$$
F^{c} \subseteq F_{2} \Rightarrow F_{2}^{c} \subseteq F
$$

and

$$
F_{1} \cap F_{2}=\varnothing \Rightarrow F_{1} \subseteq F_{2}^{c}
$$


hence

$$
G \subseteq F_{1} \subseteq F_{2}^{c} \subseteq F
$$

and since $F_{2}^{c}$ is an open subset containing $F_{1}$, we conclude that $F_{1}^{\Lambda} \subseteq F_{2}^{c}$, i.e.,

$$
G \subseteq F_{1} \subseteq F_{1}^{\Lambda} \subseteq F
$$

By setting $H=F_{1}$, condition (ii) holds.

(ii) $\Rightarrow$ (i) Suppose that $G_{1}, G_{2}$ are two disjoint subsets of $X$, such that $G_{1}$ is $\alpha$-open and $G_{2}$ is $C$-open.

This implies that $G_{2} \subseteq G_{1}^{c}$ and $G_{1}^{c}$ is an $\alpha$-closed subset of $X$. Hence by (ii) there exists a closed set $H$ such that $G_{2} \subseteq H \subseteq H^{\Lambda} \subseteq G_{1}^{c}$.

But

$$
H \subseteq H^{\Lambda} \Rightarrow H \cap\left(H^{\Lambda}\right)^{c}=\varnothing
$$

and

$$
H^{\Lambda} \subseteq G_{1}^{c} \Rightarrow G_{1} \subseteq\left(H^{\Lambda}\right)^{c}
$$

Furthermore, $\left(H^{\Lambda}\right)^{c}$ is a closed subset of $X$. Hence $G_{2} \subseteq H, G_{1} \subseteq\left(H^{\Lambda}\right)^{c}$ and $H \cap\left(H^{\Lambda}\right)^{c}=\varnothing$. This means that condition (i) holds.

Lemma 3.2. Suppose that $X$ is a topological space. If each pair of disjoint subsets $G_{1}, G_{2}$ of $X$, where $G_{1}$ is $\alpha$-open and $G_{2}$ is $C$-open, can be separated by closed subsets of $X$, then there exists a contra-continuous function $h: X \rightarrow[0,1]$ such that $h\left(G_{2}\right)=\{0\}$ and $h\left(G_{1}\right)=\{1\}$.

Proof. Suppose $G_{1}$ and $G_{2}$ are two disjoint subsets of $X$, where $G_{1}$ is $\alpha$-open and $G_{2}$ is $C$-open. Since $G_{1} \cap G_{2}=\varnothing$, hence $G_{2} \subseteq G_{1}^{c}$. In particular, since $G_{1}^{c}$ is an $\alpha$-closed subset of $X$ containing the $C$-open subset $G_{2}$ of $X$, by Lemma 3.1, there exists a closed subset $H_{1 / 2}$ such that

$$
G_{2} \subseteq H_{1 / 2} \subseteq H_{1 / 2}^{\Lambda} \subseteq G_{1}^{c}
$$


Note that $H_{1 / 2}$ is also an $\alpha$-closed subset of $X$ and contains $G_{2}$, and $G_{1}^{c}$ is an $\alpha$-closed subset of $X$ and contains the $C$-open subset $H_{1 / 2}^{\Lambda}$ of $X$. Hence, by Lemma 3.1, there exists closed subsets $H_{1 / 4}$ and $H_{3 / 4}$ such that

$$
G_{2} \subseteq H_{1 / 4} \subseteq H_{1 / 4}^{\Lambda} \subseteq H_{1 / 2} \subseteq H_{1 / 2}^{\Lambda} \subseteq H_{3 / 4} \subseteq H_{3 / 4}^{\Lambda} \subseteq G_{1}^{c}
$$

By continuing this method for every $t \in D$, where $D \subseteq[0,1]$ is the set of rational numbers that their denominators are exponents of 2 , we obtain closed subsets $H_{t}$ with the property that if $t_{1}, t_{2} \in D$ and $t_{1}<t_{2}$, then $H_{t_{1}} \subseteq H_{t_{2}}$. We define the function $h$ on $X$ by $h(x)=\inf \left\{t: x \in H_{t}\right\}$ for $x \notin G_{1}$ and $h(x)=1$ for $x \in G_{1}$.

Note that for every $x \in X, 0 \leq h(x) \leq 1$, i.e., $h$ maps $X$ into [0,1]. Also, we note that for any $t \in D, \quad G_{2} \subseteq H_{t}$; hence $h\left(G_{2}\right)=\{0\}$. Furthermore, by definition, $h\left(G_{1}\right)=\{1\}$. It remains only to prove that $h$ is a contra-continuous function on $X$. For every $\alpha \in \mathbb{R}$, we have if $\alpha \leq 0$, then $\{x \in X: h(x)<\alpha\}=\varnothing$ and if $0<\alpha$, then $\{x \in X: h(x)<\alpha\}=\bigcup\left\{H_{t}: t<\alpha\right\}$, hence, they are closed subsets of $X$. Similarly, if $\alpha<0$, then $\{x \in X: h(x)>\alpha\}=X$ and if $0 \leq \alpha$, then $\{x \in X: h(x)>\alpha\}=$ $\bigcup\left\{\left(H_{t}^{\Lambda}\right)^{c}: t>\alpha\right\}$ hence, every of them is a closed subset. Consequently $h$ is a contracontinuous function.

Lemma 3.3. Suppose that $X$ is a topological space such that every two disjoint $C$-open and $\alpha$-open subsets of $X$ can be separated by closed subsets of X. The following conditions are equivalent:

(i) Every countable covering of $C$-closed (resp. $\alpha$-closed) subsets of $X$ has a refinement consisting of $\alpha$-closed (resp. $C$-closed) subsets of $X$ such that for every $x \in X$, there exists a closed subset of $X$ containing $x$ such that it intersects only finitely many members of the refinement.

(ii) Corresponding to every decreasing sequence $\left\{G_{n}\right\}$ of C-open (resp. $\alpha$-open) subsets of $X$ with empty intersection there exists a decreasing sequence $\left\{F_{n}\right\}$ of $\alpha$-closed (resp. C-closed) subsets of $X$ such that $\bigcap_{n=1}^{\infty} F_{n}=\varnothing$ and for every $n \in \mathbb{N}, G_{n} \subseteq F_{n}$. 
Proof. (i) $\Rightarrow$ (ii) Suppose that $\left\{G_{n}\right\}$ is a decreasing sequence of $C$-open (resp. $\alpha$-open) subsets of $X$ with empty intersection. Then $\left\{G_{n}^{c}: n \in \mathbb{N}\right\}$ is a countable covering of $C$-closed (resp. $\alpha$-closed) subsets of X. By hypothesis (i) and Lemma 3.1, this covering has a refinement $\left\{V_{n}: n \in \mathbb{N}\right\}$ such that every $V_{n}$ is a closed subset of $X$ and $V_{n}^{\Lambda} \subseteq G_{n}^{c}$. By setting $F_{n}=\left(V_{n}^{\Lambda}\right)^{c}$, we obtain a decreasing sequence of closed subsets of $X$ with the required properties.

(ii) $\Rightarrow$ (i) Now if $\left\{H_{n}: n \in \mathbb{N}\right\}$ is a countable covering of $C$-closed (resp. $\alpha$-closed) subsets of $X$, we set for $n \in \mathbb{N}, G_{n}=\left(\bigcup_{i=1}^{n} H_{i}\right)^{c}$. Then $\left\{G_{n}\right\}$ is a decreasing sequence of $C$-open (resp. $\alpha$-open) subsets of $X$ with empty intersection. By (ii) there exists a decreasing sequence $\left\{F_{n}\right\}$ consisting of $\alpha$-closed (resp. $C$-closed) subsets of $X$ such that $\bigcap_{n=1}^{\infty} F_{n}=\varnothing$ and for every $n \in \mathbb{N}, G_{n} \subseteq F_{n}$. Now we define the subsets $W_{n}$ of $X$ in the following manner:

$W_{1}$ is a closed subset of $X$ such that $F_{1}^{c} \subseteq W_{1}$ and $W_{1}^{\Lambda} \cap G_{1}=\varnothing$.

$W_{2}$ is a closed subset of $X$ such that $W_{1}^{\Lambda} \cup F_{2}^{c} \subseteq W_{2}$ and $W_{2}^{\Lambda} \cap G_{2}=\varnothing$, and so on. (By Lemma 3.1, $W_{n}$ exists).

Then since $\left\{F_{n}^{c}: n \in \mathbb{N}\right\}$ is a covering for $X$, hence $\left\{W_{n}: n \in \mathbb{N}\right\}$ is a covering for $X$ consisting of closed sets. Moreover, we have

(i) $W_{n}^{\Lambda} \subseteq W_{n+1}$,

(ii) $F_{n}^{c} \subseteq W_{n}$,

(iii) $W_{n} \subseteq \bigcup_{i=1}^{n} H_{i}$.

Now setting $S_{1}=W_{1}$ and for $n \geq 2$, we set $S_{n}=W_{n+1} \backslash W_{n-1}^{\Lambda}$.

Then since $W_{n-1}^{\Lambda} \subseteq W_{n}$ and $S_{n} \supseteq W_{n+1} \backslash W_{n}$, it follows that $\left\{S_{n}: n \in \mathbb{N}\right\}$ consists of closed sets and covers $X$. Furthermore, $S_{i} \cap S_{j} \neq \varnothing$ if and only if $|i-j| \leq 1$. Finally, consider the following sets: 


$$
\begin{aligned}
& S_{1} \cap H_{1}, \quad S_{1} \cap H_{2} \\
& S_{2} \cap H_{1}, \quad S_{2} \cap H_{2}, \quad S_{2} \cap H_{3} \\
& S_{3} \cap H_{1}, \quad S_{3} \cap H_{2}, \quad S_{3} \cap H_{3}, \quad S_{3} \cap H_{4} \\
& \vdots \\
& S_{i} \cap H_{1}, \quad S_{i} \cap H_{2}, \quad S_{i} \cap H_{3}, \quad S_{i} \cap H_{4}, \quad \cdots, \quad S_{i} \cap H_{i+1}
\end{aligned}
$$

These sets are closed sets, cover $X$ and refine $\left\{H_{n}: n \in \mathbb{N}\right\}$. In addition, $S_{i} \cap H_{j}$ can intersect at most the sets in its row, immediately above, or immediately below row.

Hence if $x \in X$ and $x \in S_{n} \cap H_{m}$, then $S_{n} \cap H_{m}$ is a closed set containing $x$ that intersects at most finitely many of sets $S_{i} \cap H_{j}$. Consequently, $\left\{S_{i} \cap H_{j}: i \in \mathbb{N}, j=1, \ldots, i+1\right\}$ refines $\left\{H_{n}: n \in \mathbb{N}\right\}$ such that its elements are closed sets, and for every point in $X$ we can find a closed set containing the point that intersects only finitely many elements of that refinement.

Corollary 3.5. If every two disjoint $C$-open and $\alpha$-open subsets of $X$ can be separated by closed subsets of $X$, and in addition, every countable covering of $C$-closed (resp. $\alpha$-closed) subsets of $X$ has a refinement that consists of $\alpha$-closed (resp. $C$-closed) subsets of $X$ such that for every point of $X$ we can find a closed subset containing that point such that it intersects only a finite number of refining members then $X$ has the weakly cc-insertion property for $(c \alpha c, c C c)(\operatorname{resp} .(c C c, c \alpha c))$.

Proof. Since every two disjoint $C$-open and $\alpha$-open sets can be separated by closed subsets of $X$, therefore by Corollary 3.4, $X$ has the weak $c c$-insertion property for $(c \alpha c, c C c)$ and $(c C c, c \alpha c)$. Now suppose that $f$ and $g$ are real-valued functions on $X$ with $g<f$, such that $g$ is $c \alpha c$ (resp. $c C c$ ), $f$ is $c C c$ (resp. $c \alpha c$ ) and $f-g$ is $c C c$ (resp. $c \alpha c$ ). For every $n \in \mathbb{N}$, set

$$
A\left(f-g, 3^{-n+1}\right)=\left\{x \in X:(f-g)(x) \leq 3^{-n+1}\right\}
$$

Since $f-g$ is $c C c$ (resp. $c \alpha c$ ), hence $A\left(f-g, 3^{-n+1}\right)$ is a $C$-open (resp. $\alpha$-open) subset of $X$. Consequently, $\left\{A\left(f-g, 3^{-n+1}\right)\right\}$ is a decreasing sequence of $C$-open (resp. 
$\alpha$-open) subsets of $X$ and furthermore since $0<f-g$, it follows that $\bigcap_{n=1}^{\infty} A\left(f-g, 3^{-n+1}\right)=\varnothing$. Now by Lemma 3.3, there exists a decreasing sequence $\left\{D_{n}\right\}$ of $\alpha$-closed (resp. $C$-closed) subsets of $X$ such that $A\left(f-g, 3^{-n+1}\right) \subseteq D_{n}$ and $\bigcap_{n=1}^{\infty} D_{n}=\varnothing$. But by Lemma 3.2, the pair $A\left(f-g, 3^{-n+1}\right)$ and $X \backslash D_{n}$ of $C$-open (resp. $\alpha$-open) and $\alpha$-open (resp. $C$-open) subsets of $X$ can be completely separated by contra-continuous functions. Hence by Theorem 2.2, there exists a contra-continuous function $h$ defined on $X$ such that $g<h<f$, i.e., $X$ has the weakly $c c$-insertion property for $(c \alpha c, c C c)(\operatorname{resp} .(c C c, c \alpha c))$.

\section{References}

[1] A. Al-Omari and M. S. Md Noorani, Some properties of contra-b-continuous and almost contra-b-continuous functions, European J. Pure. Appl. Math. 2(2) (2009), 213-230.

[2] F. Brooks, Indefinite cut sets for real functions, Amer. Math. Monthly 78 (1971), 10071010. https://doi.org/10.1080/00029890.1971.11992929

[3] M. Caldas and S. Jafari, Some properties of contra- $\beta$-continuous functions, Mem. Fac. Sci. Kochi. Univ. 22 (2001), 19-28.

[4] J. Dontchev, Characterization of some peculiar topological space via $\mathcal{A}$ - and $\mathcal{B}$-sets, Acta Math. Hungar. 69(1-2) (1995), 67-71. https://doi.org/10.1007/BF01874608

[5] J. Dontchev, Contra-continuous functions and strongly S-closed space, Internat. J. Math. Math. Sci. 19(2) (1996), 303-310. https://doi.org/10.1155/S0161171296000427

[6] J. Dontchev and H. Maki, On sg-closed sets and semi- $\lambda$-closed sets, Questions Answers Gen. Topology 15(2) (1997), 259-266.

[7] J. Dontchev, Between $\alpha$ - and $\beta$-sets, Math. Balkanica (N.S) 12(3-4) (1998), 295-302.

[8] E. Ekici, On contra-continuity, Annales Univ. Sci. Bodapest 47 (2004), 127-137.

[9] E. Ekici, New forms of contra-continuity, Carpathian J. Math. 24(1) (2008), 37-45.

[10] A.I. El-Magbrabi, Some properties of contra-continuous mappings, Int. J. General Topol. 3(1-2) (2010), 55-64.

[11] M. Ganster and I. Reilly, A decomposition of continuity, Acta Math. Hungar. 56(3-4) (1990), 299-301. https://doi.org/10.1007/BF01903846 
[12] E. Hatir, T. Noiri and S. Yüksel, A decomposition of continuity, Acta Math. Hungar. 70(1-2) (1996), 145-150. https://doi.org/10.1007/BF00113919

[13] S. Jafari and T. Noiri, Contra-continuous function between topological spaces, Iranian Int. J. Sci. 2 (2001), 153-167.

[14] S. Jafari and T. Noiri, On contra-precontinuous functions, Bull. Malaysian Math. Sc. Soc. 25 (2002), 115-128.

[15] M. Katĕtov, On real-valued functions in topological spaces, Fund. Math. 38 (1951), 8591. https://doi.org/10.4064/fm-38-1-85-91

[16] M. Katětov, Correction to "On real-valued functions in topological spaces", Fund. Math. 40 (1953), 203-205. https://doi.org/10.4064/fm-40-1-203-205

[17] E. Lane, Insertion of a continuous function, Pacific J. Math. 66 (1976), 181-190. https://doi.org/10.2140/pjm.1976.66.181

[18] S. N. Maheshwari and R. Prasad, On $R_{O s}$-spaces, Portugal. Math. 34 (1975), 213-217.

[19] H. Maki, Generalized $\Lambda$-sets and the associated closure operator, The special Issue in commemoration of Prof. Kazuada Ikeda's Retirement (1986), 139-146.

[20] M. Mirmiran and B. Naderi, Strong insertion of a contra- $\alpha$-continuous function between two comparable real-valued functions, Earthline J. Math. Sci. 2(1) (2019), 223-239. https://doi.org/10.34198/ejms.2119.223239

[21] M. Mirmiran and B. Naderi, Insertion of a contra- $\alpha$-continuous function, Earthline J. Math. Sci. 2(2) (2019), 383-393. https://doi.org/10.34198/ejms.2219.383393

[22] M. Mrsevic, On pairwise $R_{0}$ and pairwise $R_{1}$ bitopological spaces, Bull. Math. Soc. Sci. Math. R. S. Roumanie 30(1986), 141-145.

[23] A. A. Nasef, Some properties of contra- $\gamma$-continuous functions, Chaos Solitons Fractals 24 (2005), 471-477. https://doi.org/10.1016/j.chaos.2003.10.033

[24] M. Przemski, A decomposition of continuity and $\alpha$-continuity, Acta Math. Hungar. 61(12) (1993), 93-98. https://doi.org/10.1007/BF01872101 